\title{
Editors' Note: Current Research on Grammar Teaching. What is the Use of Teaching Grammar?
}

Nota de les editores: Recerca actual sobre l'ensenyament de la gramàtica. Per a què serveix ensenyar gramàtica?

Nota de las editoras: Investigación actual sobre la enseñanza de la gramática. ¿Para qué sirve enseñar gramática?

Note des éditrices : Recherche actuelle sur l'enseignement de la grammaire. Pourquoi enseigner la grammaire?

Sylvie Marcotte

Université du Québec en Outaouais, campus de Saint-Jérôme

Morgane Beaumanoir-Secq

Université de Paris

Aina Reig

Universitat de València

EdITORS’ Note: CURRENT RESEARCH ON GRAMMAR TEACHING. WHAT IS THE USE OF TEACHING GRAMMAR?

\begin{abstract}
Research on grammar teaching covers a variety of topics and adopts plural perspectives. The III International Conference on Teaching Grammar (Congram19), held at the Autonomous University of Barcelona from January 23 to 25, 2019 , is a sample of this. The presence of current research, carried out in various contexts, was an opportunity to consolidate a common field and to reflect on the particularities of the research carried out in each of these contexts, clearly linked to the purposes assigned to grammar teaching. This special issue includes the contributions of 16 researchers resulting from this conference. The reader will find these contributions in two parts: the first part in the previous issue of Bellaterra Journal of Teaching \& Learning Language \& Literature (13.2) and the second part in the present issue (13.3).
\end{abstract}

Keywords: Congram19; Grammar teaching; Primary Education; Secondary Education; Higher Education 
For young researchers like us, taking part in international scientific events as the III International Conference on Teaching Grammar (Congram19), held at the Autonomous University of Barcelona from January 23 to 25, 2019, represents an excellent opportunity to know the research on teaching grammar that is currently being developed in different contexts and from plural perspectives. The Congram19 showed that, internationally, there is a common field in which it is possible to discuss grammar teaching. Events like this also allow for reflection on the specific challenges of each research and teaching context. From the scientific communications of the Congram19, as well as from the articles that result from these communications, it follows that the expectations about the study of the language are not the same in all contexts and that the purposes assigned to teaching grammar have a decisive influence on this teaching: the nature of the linguistic challenge involved in reading and writing, the social relationship with the norm, and the school tradition are parameters that influence the place of grammar in the teaching, as well as the contents and teaching strategies of grammar that are given priority.

If we place ourselves in the Francophone context, researchers in French didactics are far from a consensus on the issue of the purposes of teaching grammar. In the 1980s, Combettes (1982) noted tensions that affect the question of the objectives of language teaching and Bronckart and Besson (1988) asked: "And what if grammar were not useless?” (Et si la grammaire n'était pas inutile?). Chartrand (2012) highlights three viewpoints adopted in French didactics writings in the last 25 years: the utilitarian viewpoint, according to which grammar develops the language skills of the students, an assumption based on the postulate that grammar helps students to understand and produce oral and, above all, written discourse; the communicative viewpoint, according to which grammar is at the service of communication practices; and the disciplinary and cultural viewpoint, according to which grammar is a discipline that can be taught by itself, to know and understand the system and functioning of language. The utilitarian view is very present in the Francophone context, especially in Quebec (e.g., Nadeau \& Fisher, 2006; Vincent, Émery-Bruneau, Dezutter, Lefrançois, \& Larose, 2016).

Undoubtedly, this has to do with the fact that, in the Francophone context, respect for the rule and conventions of the written language (i.e., the absence of errors, including spelling) is given a great importance; grammar is considered to be a means of avoiding these errors. For example, Lefrançois and Brissaud (2015), who compare the language requirements of different educational jurisdictions, show that in Canada, in the English-speaking education system a student is 
considered to be fluent in written language when the mistakes he/she makes do not harm the clarity of the meaning that is conveyed through the text, while in the Quebec French-speaking education system the learner is considered to be fluent in writing when the number of linguistic mistakes he/she makes falls below a certain threshold. This threshold is, in fact, stricter in Quebec than in other French-speaking education systems (such as New Brunswick or Belgium). In the Francophone context, there is also the disciplinary and cultural viewpoint according to which the teaching of grammar does not necessarily have to be at the service of the development of students' language skills. Among others, Schneuwly (1998) considers that grammar can be taught to make students understand how language works, to make them notice its regularities and perceive it as a system; it can also be taught to develop an attitude of discovery regarding grammar itself, or even to develop cognitive skills in general. As explains Chartrand (2012), from this point of view, grammatical work allows learners to transform their relationship with language, turned into an object of analysis and, therefore, to make a step backwards, to access a different way of thinking, and to forge a grammatical culture. The teaching of grammar stimulates the intellectual and cultural development of learners.

In the Hispanic context, in the last 25 years there has been a debate about the purposes of teaching grammar (see Fontich \& García-Folgado, 2018). In Spain, in the 1990s, communicative approaches became predominant. Formal grammar ceased to be the benchmark in school and the teaching of grammar was subordinated to the teaching of the language in use. In Valencia, an innovative curriculum was developed based on socio-discursive theories and discursive genres in which grammar teaching was situated within the oral and written communicative tasks (see for example Zayas, 2020). Soon, research noted that these approaches needed to be combined with more explicit and systematic attention to the functioning of the grammar system, which would allow learners to articulate grammar knowledge into a coherent system, which would not be possible if addressing specific grammar issues in an incidental way within the communicative context. These studies, developed especially in Catalonia and Valencia, are based on the interpretation of the complex reality of the classroom and the teaching and learning processes that take place in there, and has shown the central role metalinguistic activity plays in language teaching (Camps \& Milian, 2020). The research has also highlighted the role of metalinguistic activity as a permanent source of grammar learning, generated when students write cooperatively and when they focus on specific grammar content. The research for a model for teaching has taken the form of instructional sequences for learning grammar (Milian, 2020), work projects with activities of 
observation, manipulation and analysis of linguistic forms that are closely linked within and outside the communicative flow, which focus attention on the relationship between concepts (e.g., Rodríguez-Gonzalo \& Zayas, 2017). These teaching sequences propose three different ways to learn grammar: the study of the language in use, the exploration of the concepts of the grammatical system, and the contrast between languages, or the dialects or registers of the same language (Milian, 2020).

For researchers evolving in the Francophone context in which an utilitarian (and to a lesser extent disciplinary and cultural) viewpoint prevails, attending a conference like Congram19 is an opportunity to see how grammar teaching is actualized and taught in contexts in which the objectives of this teaching are different, such as the Anglophone and Hispanic contexts. Think, for example, in the Anglophone context, of the contribution of Myhill, Watson and Newman (2020) in the first part of this special issue (issue 13.2): these researchers defend the relevance of adopting a perspective in which grammar allows to choose from a wide range of language resources and become aware of the effects of these options on the reader. In the Anglophone context, where less emphasis is placed on the norm, a perspective on teaching grammar like this, communicative, can become much more relevant than in the Francophone context. We must not forget, however, that in this context there are positions that argue that grammar must also be taught by itself. For example, Hudson (2016) considers that "If grammar is only taught when it is directly relevant to a writing task, then there is essentially no systematic teaching about grammar" (p. 293), warning that this may result in grammatical learning that is not more than "an unstructured list of unrelated items" (p. 293). For a researcher evolving in the Hispanic context, in which communicative and disciplinary viewpoints prevail, Congram19 is an opportunity to see, for example, the extent to which in the Francophone context the standard continues to occupy a prominent place in grammar teaching and that collaborative writing is also considered a way to generate metalinguistic activity among students.

With the aim of contributing to the construction of a common ground for reflection and debate on the particularities of research done in various contexts, we present this special issue of Bellaterra Journal of Teaching \& Learning Language \& Literature, with 11 contributions ( 3 guest papers by senior researchers, 6 researches by young researchers, 2 interviews and 3 reviews) resulting from the III International Conference on Teaching Grammar (Congram19). The invited professors were speakers at three of the plenary conferences of the Congram19: Debra Myhill (University of Exeter), Ignacio Bosque (Complutense University of Madrid and Real Academia Española) and Enrique Bernárdez (Complutense University of 
Madrid). Regarding the interviews, four of the researchers who took part in the two round tables organized during the congress have been interviewed: Anna Camps (Autonomous University of Barcelona), Maria Josep Cuenca (University of Valencia), Joaquim Dolz (University of Geneva) and Carmen Rodríguez-Gonzalo (University of Valencia). These are, in all cases, internationally renowned researchers who, from different perspectives, have made fundamental contributions to the field before us.

Also participating in this monograph are seven young researchers with studies that are a good example of the diversity of perspectives on research in this dynamic field: primary education (Arseneau, Giguère), secondary education (Arseneau, Marcotte, Reig, Tormo) and higher education (She Honeycutt and Sears), the contexts of L1 teaching (Catalan, Spanish, and French) and L2 teaching (Spanish), monolingual (Arseneau, Giguère, Marcotte), bilingual (Reig, Tormo) and multilingual environments (She Honeycutt and Sears), etc. The diversity of views also responds to the place where grammar is located (teaching of linguistic contents, grammar linked to the teaching of reading and writing, grammar and development of cognitive skills) and to the element of the didactic triangle: learners and learning processes (Arseneau, Reig, Tormo), teachers and knowledge and beliefs, practices, methods and teaching strategies (Giguère, Marcotte, She Honeycutt and Sears) and, finally, grammar as an object of instruction (Arseneau, Marcotte, She Honeycutt and Sears).

The contributions of this special issue show that questions about the teaching and learning of grammar are diverse, and the ways to continue the research must necessarily remain open. The reader will find these contributions in two parts: the first part, in the last issue of Bellaterra Journal of Teaching \& Learning Language \& Literature (13.2), and the second part, in the present issue (13.3).

Below we present the second part, which opens with an article by the guest professor Enrique Bernárdez (Complutense University of Madrid). The author asks about the objectives of grammar teaching and the models that prevail in school and proposes to conceive the teaching of grammar as a sociocultural activity linked to the uses ("Enseñanza de la gramática como enseñanza de una actividad sociocultural"). His reflection considers other central notions including the language in use and the standard language, in addition to pluricentrism, variety, and linguistic registers.

The issue continues with the contributions of three young researchers. Marie-Hélène Giguère ("Le cercle pédagogique: pour développer les savoirs et les 
pratiques d'enseignement en grammaire actuelle") describes the effects of a professional development device called "teaching community". The study shows that the teachers who participated in teaching communities during one year developed teaching practices and knowledge related to the current pedagogical framework for grammar teaching in French as language of instruction. Aina Reig ("Regulación metacognitiva y actividad metalingüística durante el proceso de composición escrita en colaboración a partir de la lectura de fuentes diversas") discusses a classroom intervention where students of secondary schools had to write a text in Catalan collaboratively. The analysis of the interactions and drafts shows the differences in the degree of students' reasoning and the appropriation of some of the regulation strategies provided by the instructional sequence. Finally, Rosianne Arseneau ("Comment la ponctuation se développe-t-elle chez les élèves? Une revue systématique des recherches empiriques en classe de français langue d'enseignement") examines 15 empirical studies on the development of punctuation among elementary and secondary students (6 to 17 years old) in French as a language of instruction. The author establishes three stages in the acquisition of punctuation and emphasizes the need to develop research that links didactic classroom interventions and student learning.

Finally, this issue ends with a review and an interview. Anabella L. Poggio (University of Buenos Aires) presents a volume on research carried out over the last 25 years by researchers from the Autonomous University of Barcelona, host of Congram19. This volume, edited by Anna Camps and Xavier Fontich, is published simultaneously in English (Research and teaching at the intersection: Navigating the territory of grammar and writing in the context of metalinguistic activity, Peter Lang, 2020) and in Spanish (La actividad metalingüistica como espacio de encuentro de la escritura y la gramática: Un itinerario de enseñanza e investigación en educación lingüistica, Universidad Nacional de San Juan, 2020), the latter published in a digital version with free access. As for the interview, carried out by Alba Compte ("The role of grammar teaching in learning to write / El papel de la gramática en el aprendizaje de la escritura"), Joaquim Dolz (University of Geneva) and Carmen-Gonzalo Rodríguez (University of Valencia) talk about the bridges between grammar and writing and revision as a trigger for reflection on language. Dolz and Rodríguez-Gonzalo consider that the relationship between grammar and writing cannot be constructed based on incidental work around grammar in the context of writing. They also question whether it is strictly linguistic studies that provide an adequate framework for teaching, and defend research on the action of the teacher and, more broadly, research in didactics to progress in the field. 
We hope you find this content interesting.

\section{ACKNOWLEDGMENTS}

The special editors would like to thank Xavier Fontich for his help in preparing this issue. We would also like to thank the 32 evaluators from 22 institutions in 10 countries who took part in the preparation of this monograph:

Lourdes Aguilar (Universitat Autònoma de Barcelona), Elżbieta Awramiuk (Uniwersytet w Białymstoku), Marie-Claude Boivin (Université de Montréal), Víctor Corona Villavicencio (Universitat Autònoma de Barcelona), Ana Luísa Costa (Instituto Politécnico de Setúbal), Anna Czura (Uniwersytet Wrocławski), Kristin Denham (Western Washington University), Ilse Depraetere (University of Lille), Carme Durán Rivas (Autonomous University of Barcelona), Marie-Laure Elalouf (University of Cergy-Pontoise), Santiago Fabregat Barrios (University of Jaén), Xavier Fontich (Autonomous University of Barcelona), María José García Folgado (University of Valencia), Maria Dolores Garcia-Pastor (University of Valencia), Mabel Giammatteo (University of Buenos Aires), Patrice Gourdet (University of Cergy-Pontoise), Edita Gutiérrez Rodríguez (Universidad Complutense de Madrid), Daniel Karczewski (Uniwersytet w Białymstoku), Wonki Lee (Yonsei University), Véronique Marmy Cusin (Haute École Pédagogique Fribourg), Sandra Martin-Chang (Concordia University), Beatriz Martín Marchante (Polytechnic University of Valencia), Emilee Moore (Autonomous University of Barcelona), Dave Pippin (Young Achievers Science and Math Pilot School), Abelard Zaragoza (University of Valencia), Eli Sears (Portland State University), Liane She Honeycutt (Stanly Community College), Carmen Rodríguez Gonzalo (University of Valencia), Jean-Pierre Sautot (Université Claude Bernard), Carmen Sotomayor (University of Chile), Marcial Terrádez Gurrea (University of Valencia) and François Vincent (Université du Québec en Outaouais).

\section{REFERENCES}

Bronckart, J.-P., \& Besson, M.-J. (1988). Et si la grammaire n'était pas inutile? In D. Bain (Ed.), La recherche au service de l'enseignement? (pp. 73-93). Geneva: CRPP. https://archive-ouverte.unige.ch/unige:37612

Camps, A., \& Milian, M. (2020). Metalinguistic activity in learning to write. In A. Camps, \& X. Fontich (Eds.), Research and teaching at the intersection: Navigating the territory of grammar and writing in the context of metalinguistic activity (pp. 123-144). Brussels: Peter Lang.

Chartrand, S.-G. (2012). Quelles finalités pour l'enseignement grammatical à l'école? Une analyse des points de vue des didacticiens du français depuis 25 ans. Formation et profession, 20(3), 48-59. http://dx.doi.org/10.18162/fp.2012.222 
Combettes, B. (1982). Grammaire et enseignement du français. Pratiques: linguistique, littérature, didactique, 33, 3-11. https://doi.org/10.3406/prati.1982.1227

Fontich, X., \& García-Folgado, M. J. (2018). Grammar instruction in the Hispanic area: The case of Spain with attention to empirical studies on metalinguistic activity. Contribution to the special issue Working on grammar at school in L1 education: Empirical research across linguistic regions. L1-Educational Studies in Language and Literature, 18, 1-39. https://doi.org/10.17239/L1ESLL-2018.18.04.02

Hudson, R. (2016). Grammar instruction. In C. MacArthur, S. Graham, \& J. Fitzgerald (Eds.), Handbook of writing research (pp. 288-300). New York, London: Guilford.

Lefrançois, P., \& Brissaud, C. (2015). Les exigences linguistiques de quelques systèmes scolaires: une comparaison internationale. Mesure et évaluation en éducation, 38(3), 123-151. https://doi.org/10.7202/1036701ar

Milian, M. (2020). The instructional sequence model, twenty years on: A valid model for teaching how to learn and learning how to teach. In A. Camps, \& X. Fontich (Eds.), Research and teaching at the intersection: Navigating the territory of grammar and writing in the context of metalinguistic activity (pp. 341-350). Brussels: Peter Lang.

Myhill, D., Watson, A., \& Newman, R. (2020). Thinking differently about grammar and metalinguistic understanding in writing. Bellaterra Journal of Teaching \& Learning Language \& Literature, 13(2), e870. https://doi.org/10.5565/rev/jt13.870

Nadeau, M., \& Fisher, C. (2006). La grammaire nouvelle. La comprendre et l'enseigner. Montreal: Gaëtan Morin.

Rodríguez-Gonzalo, C., \& Zayas, F. (2017). La relación entre conocimientos gramaticales y el aprendizaje de prácticas discursivas: El adjetivo como ejemplo. Caplletra, 63, 245-277. https://doi.org/10.7203/caplletra.63.10401

Schneuwly, B. (1998). Tout n'est pas métalangagier dans l'enseignement du français. La grammaire doit-elle être utile? In J. Dolz, \& J.-C. Meyer (Eds.), Activités métalangagières et enseignement du français. Actes des journées d'étude en didactique du français (Cartigny, 28 février-1 mars 1997) (pp. 267-272). Brussels: Peter Lang. https://archive-ouverte.unige.ch/unige:31420

Vincent, F., Émery-Bruneau, J., Dezutter, O., Lefrançois, P., \& Larose, F. (2016). L'enseignement de la grammaire au service du développement de compétences en lecture et en écriture : une synthèse des connaissances. Rapport de recherche. Québec: Fonds de recherche du Québec - Société et culture. http://www.frqsc.gouv.qc.ca/documents/11326/2510085/PT_VincentF_rapport_synthese-connaissances.pdf/0701273172f1-48fa-92d2-571c5d09bd2b

Zayas, F. (2020). The composition of the news. In A. Camps, \& X. Fontich (dir.), Research and teaching at the intersection: Navigating the territory of grammar and writing in the context of metalinguistic activity (p. 101-106). Brussels: Peter Lang.

\section{SYLVIE MARCOTTE}

Assistant Professor in the Department of Educational Sciences at the Université du Québec en Outaouais, she teaches the didactics of French as a first language. Her research concerns the teaching and learning of writing from elementary school to university, as well as the links between pedagogical strategies and student learning. 


\title{
MORGANE BEAUMANOIR-SECQ
}

She holds a PhD from the Université Cergy Pontoise (2016) and is a lecturer in Education Sciences (Université de Paris), she works in the field of French language teaching, focusing especially on the representations of primary school students on the syntactic system, but also on the issues raised by grammatical terminology.

morgane.beaumanoir-secq@u-paris.fr https://orcid.org/0000-0001-6528-0806

\section{AINA REIG}

She holds a PhD in Language Teaching (Universitat de València, 2020) and is a member of the GIEL research group also in this university. She has been a lecturer at the Université de Picardie Jules Verne and at the Université Sorbonne Nouvelle in Paris. Her research focuses on information management in the process of collaborative written composition.

ainareig@gmail.com https://orcid.org/0000-0001-5202-6876

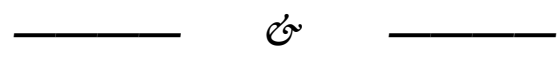

\section{NOTA DE LES EDITORES: RECERCA ACTUAL SOBRE L'ENSENYAMENT DE LA GRAMÀTICA. PER A QUÈ SERVEIX ENSENYAR GRAMÀTICA?}

\begin{abstract}
Resum: La recerca sobre l'ensenyament de la gramàtica abasta temes diversos i adopta perspectives plurals. El III Congrés Internacional sobre Ensenyament de la Gramàtica (Congram19), celebrat a la Universitat Autònoma de Barcelona del 23 al 25 de gener de 2019, n'és una mostra. La presència de treballs de recerca actuals realitzats en diversos contextos va ser una oportunitat per a contribuir a un camp comú en el qual sigui possible reflexionar i debatre sobre les particularitats de la recerca realitzada en cadascun d'aquests contextos, lligada clarament a les finalitats assignades a l'ensenyament de la gramàtica. En aquest número especial es recullen les aportacions de 16 investigadors resultants d'aquest congrés. El lector trobarà aquestes contribucions en dues parts: la primera part, en el número anterior de Bellaterra Journal of Teaching \& Learning Language \& Literature (13.2), i la segona part, en el número present (13.3).
\end{abstract}

Paraules clau: Congram19; Ensenyament de la gramàtica; Educació primària; Educació secundària; Ensenyament superior

In Memoriam: Wayne O’Neil (1931-2020)

Participar en esdeveniments científics internacionals com el III Congrés Internacional sobre Ensenyament de la Gramàtica (Congram19), celebrat a la Universitat Autònoma de Barcelona del 23 al 25 de gener de 2019, representa, per a joves investigadores com nosaltres, una excel-lent oportunitat de conèixer la recerca sobre l'ensenyament de la gramàtica que es desenvolupa actualment en diversos contextos i des de perspectives plurals. El Congram19 va evidenciar que, internacionalment, hi ha un camp comú en el qual és possible debatre sobre l'ensenyament 
de la gramàtica. Esdeveniments com aquest també permeten reflexionar sobre els reptes específics de cada context d'investigació i d'ensenyament. De les comunicacions científiques del Congram19, així com dels articles que aquestes comunicacions van originar, es desprèn que les expectatives sobre l'estudi de la llengua no són les mateixes en tots els contextos i que els propòsits atribuïts a l'ensenyament de la gramàtica tenen una influència decisiva en aquest ensenyament: la naturalesa del repte lingüístic que impliquen la lectura i l'escriptura, la relació social amb la norma i la tradició escolar són paràmetres que influeixen en el lloc que ocupa la gramàtica en l'ensenyament, així com els continguts i les estratègies d'ensenyament de la gramàtica als quals es dona prioritat.

Si ens situem en el context francòfon, els investigadors en didàctica del francès estan lluny d'un consens sobre la qüestió dels propòsits d'ensenyar gramàtica. Als anys vuitanta del segle passat, Combettes (1982) va assenyalar les tensions que afectaven la qüestió dels objectius de l'ensenyament de la llengua, i Bronckart i Besson (1988) es preguntaven: "I si la gramàtica no fos inútil?". Chartrand (2012) destaca tres punts de vista defensats en els escrits sobre la didàctica del francès durant els darrers 25 anys: el punt de vista utilitarista, segons el qual la gramàtica serveix per a desenvolupar les habilitats lingüístiques dels alumnes, que es basa en el postulat que la gramàtica ajuda els estudiants a comprendre i produir discursos orals i, sobretot, escrits; el punt de vista comunicatiu, segons el qual la gramàtica està al servei de les pràctiques de comunicació; i el punt de vista disciplinari i cultural, segons el qual la gramàtica és una disciplina que es pot ensenyar per si mateixa, per a conèixer i comprendre el sistema i el funcionament de la llengua. El punt de vista utilitarista està molt present en el context francòfon, especialment al Quebec (per exemple, Nadeau i Fisher, 2006; Vincent, Émery-Bruneau, Dezutter, Lefrançois i Larose, 2016).

Sens dubte, això té relació amb el fet que, en el context francòfon, es dona molta importància al respecte de la norma i a les convencions de la llengua escrita, és a dir, a l'absència d'errors, sobretot ortogràfics; es considera que la gramàtica és un mitjà per a evitar aquests errors. Per exemple, Lefrançois i Brissaud (2015), que comparen les exigències lingüístiques dels diferents sistemes educatius, mostren que al Canadà, en el sistema educatiu anglòfon es considera que un alumne domina la llengua escrita quan els errors que comet no perjudiquen la claredat del sentit que es comunica a través del text, mentre que en el sistema educatiu francòfon quebequès es considera que l'aprenent domina l'escriptura quan el nombre d'errors lingüístics que comet se situa per sota d'un llindar determinat. Aquest llindar és, de fet, més estricte al Quebec que en altres sistemes educatius francòfons (com ara 
Nova Brunsvic o Bèlgica). En el context francòfon, també hi ha el punt de vista disciplinari i cultural segons el qual l'ensenyament de la gramàtica no ha d'estar necessàriament al servei del desenvolupament de les habilitats lingüístiques dels alumnes. Entre d'altres, Schneuwly (1998) considera que la gramàtica es pot ensenyar per a fer comprendre com funciona la llengua, donar-ne a conèixer la regularitat i fer que es percebi com un sistema; també es pot ensenyar per a desenvolupar una actitud de descoberta respecte de la gramàtica mateixa, o fins i tot per a desenvolupar habilitats cognitives en general. Tal com ho explica Chartrand (2012), des d'aquest punt de vista, el treball gramatical permet als aprenents transformar la seva relació amb la llengua, convertida en un objecte d'anàlisi i, per tant, distanciarse'n per a accedir a una manera diferent de pensar i de forjar una cultura gramatical. L'ensenyament de la gramàtica estimula el desenvolupament intel-lectual i cultural dels aprenents.

En el context hispànic, en els darrers 25 anys s'ha desenvolupat un debat sobre les finalitats de l'ensenyament de la gramàtica (vegeu Fontich i García-Folgado, 2018). A l'Estat espanyol, als anys noranta, van adquirir preeminència els enfocaments comunicatius. La gramàtica formal va deixar de ser el referent a l'escola i l'ensenyament de la gramàtica es va supeditar a l'ensenyament de la llengua en ús. Al País Valencià, es va desenvolupar un innovador currículum basat en les teories sociodiscursives i els gèneres discursius en el qual l'ensenyament gramatical se situava al si de les tasques comunicatives orals i escrites, de lectura i d'escriptura (vegeu per exemple Zayas, 2020). Ben aviat, la recerca va assenyalar que aquests plantejaments havien de combinar-se amb una atenció més explícita i sistemàtica al funcionament del sistema gramatical, que permetés als aprenents articular els coneixements gramaticals en un sistema coherent, la qual cosa probablement no seria possible si els sabers gramaticals específics s'abordaven d'una manera incidental en el context comunicatiu. Aquests estudis, desenvolupats especialment a Catalunya i al País Valencià, es basen en la interpretació de la realitat complexa de l'aula i dels processos d'ensenyament i aprenentatge que hi tenen lloc, i han demostrat el paper central de l'activitat metalingüística en l'ensenyament de la llengua (Camps i Milian, 2020). La recerca esmentada ha evidenciat, a més, el paper de l'activitat metalingüística com a font inesgotable d'aprenentatge gramatical que es genera quan els alumnes escriuen cooperativament i quan se centren en continguts gramaticals concrets. La cerca d'un model per a l'ensenyament s'ha concretat en les seqüències didàctiques per a aprendre gramàtica (Milian, 2020), uns projectes de treball amb activitats d'observació, manipulació i anàlisi de formes lingüístiques que es realitzen tant vinculades als usos com fora del flux comunicatiu, que centren 
l'atenció en la relació entre conceptes (vegeu per exemple Rodríguez-Gonzalo i Zayas, 2017). Aquestes seqüències d'ensenyament proposen tres camins diferents per a aprendre gramàtica: l'estudi de la llengua en ús, l'exploració dels conceptes del sistema gramatical i el contrast entre les llengües, o els dialectes o registres d'una mateixa llengua (Milian, 2020).

Per a investigadores formades en el context francòfon, en el qual prevalen perspectives utilitaristes $i$, en menor mesura, disciplinàries i culturals, assistir a un congrés com el Congram19 és una oportunitat per a veure com s'actualitza i s'ensenya la gramàtica en contextos en què els objectius d'aquest ensenyament són diferents, com ara els contextos anglòfon i hispànic. Pensem per exemple, en el context anglòfon, en la contribució de Myhill, Watson i Newman (2020), en la primera part d'aquest número especial (número 13.2): aquestes investigadores defensen la rellevància de situar-se en una perspectiva segons la qual la gramàtica permet triar entre una àmplia gamma de recursos lingüístics i prendre consciència dels efectes d'aquestes opcions sobre el lector. En el context anglòfon, en què es fa menys èmfasi en el respecte de la norma, una perspectiva d'ensenyament de la gramàtica com aquesta, comunicativa, pot esdevenir molt més rellevant que en un context francòfon. No podem oblidar, però, que en aquest context hi ha posicions que defensen que la gramàtica s'ha d'abordar també per ella mateixa. Per exemple, Hudson (2016) considera que "If grammar is only taught when it is directly relevant to a writing task, then there is essentially no systematic teaching about grammar» (p. 293), alertant que això pot tenir com a conseqüència que els aprenentatges gramaticals es converteixin en «an unstructured list of unrelated items» (p. 293). Per a una investigadora que treballa en el context hispànic, en el qual dominen les perspectives comunicativa i disciplinària, el Congram19 és una oportunitat per a veure, per exemple, que en el context francòfon, l'estàndard continua ocupant un lloc predominant en l'ensenyament gramatical i que l'escriptura col-laborativa també es considera una manera de generar activitat metalingüística entre els estudiants.

Amb l'objectiu de contribuir a la construcció d'un terreny comú per a la reflexió i el debat sobre les particularitats de la recerca feta en diversos contextos, presentem aquest número especial de Bellaterra Journal of Teaching \& Learning Language \& Literature, amb 11 contribucions (3 articles invitats d'investigadors sènior, 6 recerques de joves investigadors, 2 entrevistes i 3 ressenyes) provinents del III Congrés Internacional sobre Ensenyament de la Gramàtica (Congram19). Els professors invitats van ser ponents de tres de les conferències plenàries del Congram19: Debra Myhill (Universitat d'Exeter), Ignacio Bosque (Universitat Complutense de Madrid i Real Academia Española) i Enrique Bernárdez (Universitat 
Complutense de Madrid). Pel que fa a les entrevistes, s'han fet a quatre dels investigadors que van participar en les dues taules rodones organitzades durant el congrés: Anna Camps (Universitat Autònoma de Barcelona), Maria Josep Cuenca (Universitat de València), Joaquim Dolz (Universitat de Ginebra) i Carmen Rodríguez-Gonzalo (Universitat de València). Es tracta, en tots els casos, d'investigadors de referència internacional que des de perspectives diferents han realitzat aportacions fonamentals al camp que ens ocupa.

Així mateix, participen en aquest monogràfic set joves investigadors amb estudis que són una bona mostra de la diversitat de mirades de la recerca sobre aquest camp tan dinàmic: l'educació primària (Arseneau, Giguère), secundària (Arseneau, Marcotte, Reig, Tormo) i universitària (She Honeycutt i Sears), els contextos d'ensenyament de la L1 (català, espanyol i francès) o de la L2 (espanyol), els entorns monolingües (Arseneau, Giguère, Marcotte), bilingües (Reig, Tormo) i plurilingües (She Honeycutt i Sears), etc. La diversitat de mirades també respon al lloc en què se situa la gramàtica (ensenyament dels continguts lingüístics, gramàtica vinculada a l'ensenyament de la lectura i l'escriptura, gramàtica i desenvolupament de les habilitats cognitives) i en l'element del triangle didàctic que s'explora: els aprenents i els processos d'aprenentatge (Arseneau, Reig, Tormo), els docents i els sabers i creences, pràctiques, mètodes i estratègies d'ensenyament (Giguère, Marcotte, She Honeycutt i Sears) i, finalment, la gramàtica com a objecte d'ensenyament (Arseneau, Marcotte, She Honeycutt i Sears).

Les contribucions d'aquest número especial mostren que les preguntes sobre l'ensenyament i l'aprenentatge de la gramàtica són diverses, i que les vies per a continuar la recerca han de romandre necessàriament obertes. El lector trobarà aquestes contribucions en dues parts: la primera part, en el darrer número de $B e$ llaterra Journal of Teaching \& Learning Language \& Literature (13.2), i la segona part, en el número present (13.3).

Tot seguit presentem la segona part del monogràfic, que s'obre amb un article del professor invitat, Enrique Bernárdez (Universitat Complutense de Madrid). L'autor es pregunta pels objectius de l'ensenyament de la gramàtica i els models que prevalen a l'escola i proposa concebre l'ensenyament de la gramàtica com a activitat sociocultural lligada als usos ("Enseñanza de la gramática como enseñanza de una actividad sociocultural"). La seva reflexió té en compte altres nocions centrals entre les quals hi ha la llengua en ús i la llengua estàndard, a més del pluricentrisme, les varietats i els registres lingüístics. 
El número segueix amb les contribucions de tres joves investigadores. MarieHélène Giguère ("Le cercle pédagogique: pour développer les savoirs et les pratiques d'enseignement en grammaire actuelle") descriu els efectes d'un dispositiu de formació professional per a ensenyar francès anomenat "El cercle pedagògic". El treball demostra que els docents que van participar en aquesta formació d'un any de durada van desenvolupar pràctiques pedagògiques i coneixements lligats a la gramàtica i al seu ensenyament. Aina Reig ("Regulación metacognitiva y actividad metalingüística durante el proceso de composición escrita en colaboración a partir de la lectura de fuentes diversas”) analitza una intervenció d'aula en què els alumnes de secundària havien d'escriure un text en català de manera col-laborativa. L'anàlisi de les interaccions i dels esborranys mostra les diferències en el grau de raonament dels alumnes i també l'apropiació d'algunes de les estratègies de regulació dels continguts previstos en la intervenció. Finalment, Rosianne Arseneau ( “Comment la ponctuation se développe-t-elle chez les élèves? Une revue systématique des recherches empiriques en classe de français langue d'enseignement”) examina 15 estudis empírics de l'àmbit francòfon sobre el desenvolupament de la puntuació en primària i secundària. L'autora estableix tres etapes en l'adquisició de la puntuació i subratlla la necessitat de desenvolupar recerques que relacionin les intervencions d'ensenyament en context d'aula i els aprenentatges dels alumnes.

Finalment, aquest número acaba amb una ressenya i una entrevista. Anabella L. Poggio (Universitat de Buenos Aires) presenta un volum sobre la recerca portada a terme els darrers 25 anys per part d'investigadors de la Universitat Autònoma de Barcelona, amfitriona del Congram19. Aquest volum, editat per Anna Camps i Xavier Fontich, es publica simultàniament en versió anglesa (Research and teaching at the intersection: Navigating the territory of grammar and writing in the context of metalinguistic activity, Peter Lang, 2020) i en versió espanyola (La actividad metalingüística como espacio de encuentro de la escritura y la gramática: Un itinerario de enseñanza e investigación en educación lingüística, Universidad Nacional de San Juan, 2020), aquesta darrera publicada en versió digital d'accés lliure. Pel que fa a l'entrevista, a càrrec d'Alba Compte ( "The role of grammar teaching in learning to write / El papel de la gramática en el aprendizaje de la escritura”), Joaquim Dolz (Universitat de Ginebra) i Carmen Rodríguez-Gonzalo (Universitat de València) parlen dels ponts entre la gramàtica i l'escriptura i de la revisió com a activadora de la reflexió sobre la llengua. Dolz i Rodríguez-Gonzalo consideren que la relació entre la gramàtica i l'escriptura no pot construir-se sobre la base d'un treball incidental al voltant de la gramàtica en el context d'escriptura. Qüestionen, així mateix, que siguin els estudis estrictament lingüístics els que aportin un marc 
adequat per a l'ensenyament, i defensen l'acció del docent i, més àmpliament, la recerca en didàctica com a àmbit en el qual avançar.

Esperem que trobeu interessant aquest contingut.

\section{AgRAÏMENTS}

Les editores volen donar les gràcies a Xavier Fontich per l'ajuda durant la preparació d'aquest número especial. Igualment, també volen mostrar agraïment als 32 avaluadors i avaluadores de 22 institucions de 10 països que han participat en la preparació d'aquest monogràfic:

Lourdes Aguilar (Universitat Autònoma de Barcelona), Elżbieta Awramiuk (Uniwersytet w Białymstoku), Marie-Claude Boivin (Université de Montréal), Víctor Corona Villavicencio (Universitat Autònoma de Barcelona), Ana Luísa Costa (Instituto Politécnico de Setúbal), Anna Czura (Uniwersytet Wrocławski), Kristin Denham (Western Washington University), Ilse Depraetere (Université de Lille), Carme Durán Rivas (Universitat Autònoma de Barcelona), Marie-Laure Elalouf (Université de Cergy-Pontoise), Santiago Fabregat Barrios (Universidad de Jaén), Xavier Fontich (Universitat Autònoma de Barcelona), María José García Folgado (Universitat de València), Maria Dolores Garcia-Pastor (Universitat de València), Mabel Giammatteo (Universidad de Buenos Aires), Patrice Gourdet (Université de Cergy-Pontoise), Edita Gutiérrez Rodríguez (Universidad Complutense de Madrid), Daniel Karczewski (Uniwersytet w Białymstoku), Wonki Lee (Yonsei University), Véronique Marmy Cusin (Haute École Pédagogique Fribourg), Sandra Martin-Chang (Concordia University), Beatriz Martín Marchante (Universitat Politècnica de València), Emilee Moore (Universitat Autònoma de Barcelona), Dave Pippin (Young Achievers Science and Math Pilot School), Abelard Saragossà (Universitat de València), Eli Sears (Portland State University), Liane She Honeycutt (Stanly Community College), Carmen Rodríguez Gonzalo (Universitat de València), Jean-Pierre Sautot (Université Claude Bernard), Carmen Sotomayor (Universidad de Chile), Marcial Terrádez Gurrea (Universitat de València) i François Vincent (Université du Québec en Outaouais).

\section{REFERÈNCIES}

Bronckart, J.-P. i Besson, M.-J. (1988). Et si la grammaire n'était pas inutile? En D. Bain (Ed.), La recherche au service de l'enseignement? (pp. 73-93). Ginebra: CRPP. https://archiveouverte.unige.ch/unige:37612 
Camps, A. i Milian, M. (2020). Metalinguistic activity in learning to write. En A. Camps i X. Fontich (Eds.), Research and teaching at the intersection: Navigating the territory of grammar and writing in the context of metalinguistic activity (pp. 123-144). Brussel-les: Peter Lang.

Chartrand, S.-G. (2012). Quelles finalités pour l'enseignement grammatical à l'école? Une analyse des points de vue des didacticiens du français depuis 25 ans. Formation et profession, 20(3), 48-59. http://dx.doi.org/10.18162/fp.2012.222

Combettes, B. (1982). Grammaire et enseignement du français. Pratiques: linguistique, littérature, didactique, 33, 3-11. https://doi.org/10.3406/prati.1982.1227

Fontich, X. i García-Folgado, M. J. (2018). Grammar instruction in the Hispanic area: The case of Spain with attention to empirical studies on metalinguistic activity. Contribution to the special issue Working on grammar at school in L1 education: Empirical research across linguistic regions. L1-Educational Studies in Language and Literature, 18, 1-39. https://doi.org/10.17239/L1ESLL-2018.18.04.02

Hudson, R. (2016). Grammar instruction. En C. MacArthur, S. Graham i J. Fitzgerald (Eds.), Handbook of writing research (pp. 288-300). Nova York, Londres: Guilford.

Lefrançois, P. i Brissaud, C. (2015). Les exigences linguistiques de quelques systèmes scolaires: une comparaison internationale. Mesure et évaluation en éducation, 38(3), 123-151. https://doi.org/10.7202/1036701ar

Milian, M. (2020). The instructional sequence model, twenty years on: A valid model for teaching how to learn and learning how to teach. En A. Camps i X. Fontich (Eds.), Research and teaching at the intersection: Navigating the territory of grammar and writing in the context of metalinguistic activity (pp. 341-350). Brussel-les: Peter Lang.

Myhill, D., Watson, A. i Newman, R. (2020). Thinking differently about grammar and metalinguistic understanding in writing. Bellaterra Journal of Teaching \& Learning Language \& Literature, 13(2), e870. https://doi.org/10.5565/rev/jt13.870

Nadeau, M. i Fisher, C. (2006). La grammaire nouvelle. La comprendre et l'enseigner. Montreal: Gaëtan Morin.

Rodríguez-Gonzalo, C. i Zayas, F. (2017). La relación entre conocimientos gramaticales y el aprendizaje de prácticas discursivas: El adjetivo como ejemplo. Caplletra, 63, 245-277. https://doi.org/10.7203/caplletra.63.10401

Schneuwly, B. (1998). Tout n'est pas métalangagier dans l'enseignement du français. La grammaire doit-elle être utile? En J. Dolz i J.-C. Meyer (Eds.), Activités métalangagières et enseignement du français. Actes des journées d'étude en didactique du français (Cartigny, 28 février-1 mars 1997) (pp. 267-272). Brusel-les: Peter Lang.

https://archive-ouverte.unige.ch/unige:31420

Vincent, F., Émery-Bruneau, J., Dezutter, O., Lefrançois, P. i Larose, F. (2016). L'enseignement de la grammaire au service du développement de compétences en lecture et en écriture : une synthèse des connaissances. Rapport de recherche. Québec: Fonds de recherche du Québec - Société et culture. http://www.frqsc.gouv.qc.ca/documents/11326/2510085/PT_VincentF_rapport_synthese-connaissances.pdf/07012731$72 \mathrm{f} 1-48 \mathrm{fa}-92 \mathrm{~d} 2-571 \mathrm{c} 5 \mathrm{~d} 09 \mathrm{bd} 2 \mathrm{~b}$

Zayas, F. (2020). The composition of the news. Dins A. Camps et X. Fontich (ed.), Research and teaching at the intersection: Navigating the territory of grammar and writing in the context of metalinguistic activity (p. 101-106). Brussel-les: Peter Lang. 


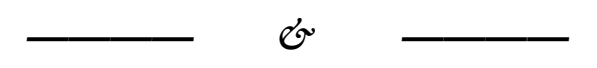

\section{SYLVIE MARCOTTE}

Professora del Departament de Ciències de l'Educació de la Université du Québec en Outaouais, ensenya didàctica del francès com a primera llengua. La seva recerca tracta sobre l'ensenyament $i$ l'aprenentatge de l'escriptura des de l'escola primària fins a la universitat, així com els vincles entre estratègies pedagògiques i aprenentatge dels alumnes.

sylvie.marcotte@uqo.ca https://orcid.org/0000-0002-6623-6169

\section{MORgane BEAUMANOIR-SECQ}

Doctora en Ciències del Llenguatge (Université de Cergy Pontoise, 2016) i professora de Ciències de l'Educació (Université de Paris), treballa en el camp de la didàctica del francès, incidint en les representacions dels estudiants de primària sobre el sistema sintàctic i en les qüestions plantejades per la terminologia gramatical.

morgane.beaumanoir-secq@u-paris.fr https://orcid.org/0000-0001-6528-0806

\section{AINA REIG}

Doctora en Didàctica de la Llengua per la Universitat de València (2020) i membre del grup de recerca GIEL d'aquesta mateixa universitat. Ha estat docent a la Université de Picardie Jules Verne i a la Université Sorbonne Nouvelle de París. La seva línia de recerca se centra en la gestió informativa en el procés de composició escrita en col-laboració.

ainareig@gmail.com https://orcid.org/0000-0001-5202-6876

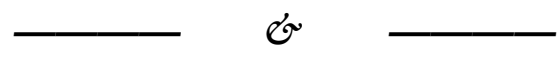

\section{NOTA DE LAS EDITORAS: INVESTIGACIÓN ACTUAL SOBRE LA ENSEÑANZA DE LA GRAMÁTICA. ¿PARA QUÉ SIRVE ENSEÑAR GRAMÁTICA?}

Resumen: La investigación sobre la enseñanza de la gramática abarca temas diversos y adopta perspectivas plurales. El III Congreso Internacional sobre Enseñanza de la Gramática (Congram19), celebrado en la Universidad Autónoma de Barcelona del 23 al 25 de enero de 2019, es una muestra de ello. La presencia de trabajos de investigación actuales realizados en diversos contextos fue una oportunidad para contribuir a un campo común en el que sea posible reflexionar y debatir sobre las particularidades de la investigación realizada en cada uno de estos contextos, ligada claramente a los fines asignados a la enseñanza de la gramática. En este número especial se recogen las aportaciones de 16 investigadores resultantes de este congreso. El lector encontrará estas contribuciones en dos partes: la primera parte, en el número anterior de Bellaterra Journal of Teaching \& Learning Language \& Literature (13.2), y la segunda parte, en el número presente (13.3).

Palabras clave: Congram19; Enseñanza de la gramática; Educación primaria; Educación secundaria; Enseñanza superior 
Participar en eventos científicos internacionales como el III Congreso Internacional sobre Enseñanza de la Gramática (Congram19), celebrado en la Universidad Autónoma de Barcelona del 23 al 25 de enero de 2019, representa, para jóvenes investigadoras como nosotras, una excelente oportunidad para conocer la investigación sobre la enseñanza de la gramática que se desarrolla actualmente en diversos contextos y desde perspectivas plurales. El Congram19 evidenció que, internacionalmente, hay un campo común en el que es posible debatir sobre la enseñanza de la gramática. Eventos como este también permiten reflexionar sobre los retos específicos de cada contexto de investigación y enseñanza. De las comunicaciones científicas del Congram19, así como de los artículos que estas comunicaciones originaron, se desprende que las expectativas sobre el estudio de la lengua no son las mismas en todos los contextos y que los propósitos atribuidos a la enseñanza de la gramática tienen una influencia decisiva en esta enseñanza: la naturaleza del reto lingüístico que implican la lectura y la escritura, la relación social con la norma y la tradición escolar son parámetros que influyen en el lugar que ocupa la gramática en la enseñanza, así como los contenidos y las estrategias de enseñanza de la gramática a los que se da prioridad.

Si nos situamos en el contexto francófono, los investigadores en didáctica del francés están lejos de un consenso sobre la cuestión de los propósitos de enseñar gramática. En los años ochenta del siglo pasado, Combettes (1982) señaló las tensiones que afectaban a la cuestión de los objetivos de la enseñanza de la lengua, y Bronckart y Besson (1988) se preguntaron: “¿Y si la gramática no fuera inútil?”. Chartrand (2012) destaca tres puntos de vista defendidos en los escritos sobre la didáctica del francés durante los últimos 25 años: el punto de vista utilitarista, según el cual la gramática sirve para desarrollar las habilidades lingüísticas de los alumnos, que se basa en el postulado que la gramática ayuda a los estudiantes a comprender y producir discursos orales y, sobre todo, escritos; el punto de vista comunicativo, según el cual la gramática está al servicio de las prácticas de comunicación; y el punto de vista disciplinario y cultural, según el cual la gramática es una disciplina que se puede enseñar por sí misma, para conocer y comprender el sistema y el funcionamiento de la lengua. El punto de vista utilitarista está muy presente en el contexto francófono, especialmente en Quebec (por ejemplo, Nadeau y Fisher, 2006; Vincent, Émery-Bruneau, Dezutter, Lefrançois y Larose, 2016).

Sin duda, esto está relacionado con el hecho de que, en el contexto francófono, se da mucha importancia al respeto a la norma y a las convenciones de la lengua escrita, es decir, a la ausencia de errores, sobre todo ortográficos, y se 
considera que la gramática sirve para evitar estos errores. Por ejemplo, Lefrançois y Brissaud (2015), que comparan las exigencias lingüísticas de los diferentes sistemas educativos, muestran que en Canadá, en el sistema educativo anglófono se considera que un alumno domina la lengua escrita cuando los errores que comete no perjudican la claridad del sentido que se comunica a través del texto, mientras que en el sistema educativo francófono quebequés se considera que el aprendiz domina la escritura cuando el número de errores lingüísticos que comete se sitúa por debajo de un umbral determinado. Este umbral es, de hecho, más estricto en Quebec que en otros sistemas educativos francófonos (como Nueva Brunswick o Bélgica). En el contexto francófono, también encontramos el punto de vista disciplinario y cultural según el cual la enseñanza de la gramática no debe estar necesariamente al servicio del desarrollo de las habilidades lingüísticas de los alumnos. Entre otros, Schneuwly (1998) considera que la gramática se puede enseñar para hacer comprender cómo funciona la lengua, dar a conocer su regularidad y hacer que esta se perciba como un sistema; también se puede enseñar para desarrollar una actitud de descubrimiento respecto de la gramática, o incluso para para desarrollar habilidades cognitivas en general. Tal como explica Chartrand (2012), desde este punto de vista, el trabajo gramatical permite a los aprendices transformar su relación con la lengua, una vez convertida en un objeto de análisis y, por tanto, distanciarse para acceder a una manera diferente de pensar y de forjar una cultura gramatical. La enseñanza de la gramática estimula el desarrollo intelectual y cultural de los aprendices.

En el contexto hispánico, en los últimos 25 años se ha desarrollado un debate sobre las finalidades de la enseñanza de la gramática (véase Fontich y GarcíaFolgado, 2018). En España, en los años noventa, adquirieron preeminencia los enfoques comunicativos. La gramática formal dejó de ser el referente en la escuela y la enseñanza de la gramática se supeditó a la enseñanza de la lengua en uso. En el País Valenciano, se desarrolló un innovador currículo basado en las teorías sociodiscursivas y los géneros discursivos en el que la enseñanza gramatical se situaba en el seno de las tareas comunicativas orales y escritas, de lectura y de escritura (véase por ejemplo Zayas, 2020). Muy pronto, la investigación señaló que estos planteamientos debían combinarse con una atención más explícita y sistemática al funcionamiento del sistema gramatical, que permitiera a los aprendices articular los conocimientos gramaticales en un sistema coherente, lo cual probablemente no sería posible si los saberes gramaticales específicos se abordasen de una manera incidental en el contexto comunicativo. Estos estudios, desarrollados especialmente en Cataluña y en Valencia, se basan en la interpretación de la realidad compleja del 
aula y de los procesos de enseñanza y aprendizaje que tienen lugar en ella, y han mostrado el papel central de la actividad metalingüística en la enseñanza de la lengua (Camps y Milian, 2020). Dicha investigación ha evidenciado, además, el papel de la actividad metalingüística como fuente inagotable de aprendizaje gramatical que se genera cuando los alumnos escriben cooperativamente y cuando se centran en contenidos gramaticales concretos. La búsqueda de un modelo para la enseñanza se ha concretado en las secuencias didácticas para aprender gramática (Milian, 2020), unos proyectos de trabajo con actividades de observación, manipulación y análisis de formas lingüísticas que se llevan a cabo vinculadas tanto a los usos como fuera del flujo comunicativo, que centran la atención en la relación entre conceptos (véase por ejemplo Rodríguez-Gonzalo y Zayas, 2017). Estas secuencias de enseñanza proponen tres caminos diferentes para aprender gramática: el estudio de la lengua en uso, la exploración de los conceptos del sistema gramatical y el contraste entre las lenguas, o los dialectos o registros de una misma lengua (Milian, 2020).

Para investigadoras formadas en el contexto francófono, en el que prevalecen perspectivas utilitaristas y, en menor medida, disciplinarias y culturales, asistir a un congreso como el Congram19 es una oportunidad para ver cómo se actualiza y se enseña la gramática en contextos en los que los objetivos de esta enseñanza son diferentes, como los contextos anglófono e hispánico. Pensemos, por ejemplo, en el contexto anglófono, en la contribución de Myhill, Watson y Newman (2020), de la primera parte de este número especial (número 13.2): estas investigadoras defienden la relevancia de situarse en una perspectiva según la cual la gramática permite elegir entre una amplia gama de recursos lingüísticos y tomar conciencia de los efectos de estas opciones sobre el lector. En el contexto anglófono, en el que se hace menos énfasis en el respeto a la norma, una perspectiva de enseñanza de la gramática como esta, comunicativa, puede ser mucho más relevante que en un contexto francófono. No podemos olvidar, sin embargo, que en este contexto hay posiciones que defienden que la gramática debe abordarse también por ella misma. Por ejemplo, Hudson (2016) considera que «If grammar is only taught when it is directly relevant to a writing task, then there is essentially no systematic teaching about grammar» (p. 293), alertando de que esto puede tener como consecuencia que los aprendizajes gramaticales se conviertan en «an unstructured list of unrelated items» (p. 293). Para una investigadora que trabaja en el contexto hispánico, en el que dominan las perspectivas comunicativa y disciplinaria, el Congram19 es una oportunidad para ver, por ejemplo, que en el contexto francófono, el estándar sigue ocupando un lugar predominante en la enseñanza gramatical y que la 
escritura colaborativa también se considera una forma de generar actividad metalingüística entre los estudiantes.

Con el objetivo de contribuir a la construcción de un terreno común para la reflexión y el debate sobre las particularidades de la investigación realizada en diversos contextos, presentamos este número especial de Bellaterra Journal of Teaching \& Learning Language \& Literature, con 11 contribuciones (3 artículos invitados de investigadores sénior, 6 estudios de jóvenes investigadores, 2 entrevistas y 3 reseñas) provenientes del III Congreso Internacional sobre Enseñanza de la Gramática (Congram19). Los profesores invitados fueron ponentes de tres de las conferencias plenarias del Congram19: Debra Myhill (Universidad de Exeter), Ignacio Bosque (Universidad Complutense de Madrid y Real Academia Española) y Enrique Bernárdez (Universidad Complutense de Madrid). En cuanto a las entrevistas, se han hecho a cuatro de los investigadores que participaron en las dos mesas redondas organizadas durante el congreso: Anna Camps (Universidad Autónoma de Barcelona), Maria Josep Cuenca (Universidad de Valencia), Joaquim Dolz (Universidad de Ginebra) y Carmen Rodríguez-Gonzalo (Universidad de Valencia). Se trata, en todos los casos, de investigadores de referencia internacional que desde perspectivas diferentes han realizado aportaciones fundamentales en el campo que nos ocupa.

Asimismo, participan en este monográfico siete jóvenes investigadores con estudios que son una buena muestra de la diversidad de miradas de la investigación sobre este campo tan dinámico: Educación Primaria (Arseneau, Giguère), Educación Secundaria (Arseneau, Marcotte, Reig, Tormo) y Educación Superior (She Honeycutt y Sears), contextos de enseñanza de la L1 (catalán, español y francés) o de la L2 (español), los entornos monolingües (Arseneau, Giguère, Marcotte), bilingües (Reig, Tormo) y plurilingües (She Honeycutt y Sears), etc. La diversidad de miradas también responde al lugar donde se sitúa la gramática (enseñanza de los contenidos lingüísticos, gramática vinculada a la enseñanza de la lectura y la escritura, gramática y desarrollo de las habilidades cognitivas) y en el elemento del triángulo didáctico que se explora: los aprendices y los procesos de aprendizaje (Arseneau, Reig, Tormo), los docentes y los saberes y creencias, prácticas, métodos y estrategias de enseñanza (Giguère, Marcotte, She Honeycutt y Sears) y, finalmente, la gramática como objeto de enseñanza (Arseneau, Marcotte, She Honeycutt y Sears).

Las contribuciones de este número especial muestran que las preguntas sobre la enseñanza y el aprendizaje de la gramática son diversas, y que las vías para continuar la búsqueda deben permanecer necesariamente abiertas. El lector 
encontrará estas aportaciones en dos partes: la primera parte, en el número anterior de Bellaterra Journal of Teaching \& Learning Language \& Literature (13.2), y la segunda parte, en el presente número (13.3).

A continuación, presentamos la segunda parte del monográfico, que se abre con un artículo del profesor invitado, Enrique Bernárdez (Universidad Complutense de Madrid). El autor se pregunta por los objetivos de la enseñanza de la gramática y los modelos que prevalecen en la escuela y propone concebir la enseñanza de la gramática como actividad sociocultural ligada a los usos ("Enseñanza de la gramática como enseñanza de una actividad sociocultural”). Su reflexión tiene en cuenta otras nociones centrales entre las que encontramos la lengua en uso y la lengua estándar, además del pluricentrismo, las variedades y los registros lingüísticos.

El número sigue con las contribuciones de tres jóvenes investigadoras. Marie-Hélène Giguère ("Le cercle pédagogique: pour développer les savoirs et les pratiques d'enseignement en grammaire actuelle") describe los efectos de un dispositivo de formación profesional llamado "El círculo pedagógico". El trabajo muestra que los docentes que participaron en esta formación de un año de duración desarrollaron prácticas pedagógicas y conocimientos ligados al marco pedagógico actual para la enseñanza de la gramática en francés como idioma de escolarización. Aina Reig ("Regulación metacognitiva y actividad metalingüística durante el proceso de composición escrita en colaboración a partir de la lectura de fuentes diversas") analiza una intervención de aula en la que los alumnos de secundaria debían escribir colaborativamente un texto en catalán. El análisis de las interacciones y de los borradores muestra las diferencias en el grado de razonamiento de los alumnos y también la apropiación de algunas de las estrategias de regulación de los contenidos previstos en la intervención. Finalmente, Rosianne Arseneau ("Comment la ponctuation se développe-t-elle chez les élèves? Une revue systématique des recherches empiriques en classe de français langue d'enseignement”) examina 15 estudios empíricos del ámbito francófono sobre el desarrollo de la puntuación en Primaria y Secundaria. La autora establece tres etapas en la adquisición de la puntuación y subraya la necesidad de desarrollar investigaciones que relacionen las intervenciones didácticas en contexto de aula y los aprendizajes de los alumnos.

Finalmente, este número termina con una reseña y una entrevista. Anabella L. Poggio (Universidad de Buenos Aires) presenta un volumen sobre la investigación llevada a cabo en los últimos 25 años por parte de investigadores de la Universidad Autónoma de Barcelona, anfitriona del Congram19. Este volumen, 
editado por Anna Camps y Xavier Fontich, se publica simultáneamente en versión inglesa (Research and teaching at the intersection: Navigating the territory of grammar and writing in the context of metalinguistic activity, Peter Lang, 2020) y en versión española (La actividad metalingüística como espacio de encuentro de la escritura y la gramática: Un itinerario de enseñanza e investigación en educación lingüística, Universidad Nacional de San Juan, 2020), esta última publicada en versión digital de acceso libre. En relación con la entrevista, a cargo de Alba Compte ("The role of grammar teaching in learning to write / El papel de la gramática en el aprendizaje de la escritura"), Joaquim Dolz (Universidad de Ginebra) y Carmen Rodríguez-Gonzalo (Universidad de Valencia) hablan de los puentes entre la gramática y la escritura y de la revisión como activadora de la reflexión sobre la lengua. Dolz y Rodríguez-Gonzalo consideran que la relación entre la gramática y la escritura no puede construirse sobre la base de un trabajo incidental acerca de la gramática en el contexto de escritura. Cuestionan, asimismo, que sean los estudios estrictamente lingüísticos los que aporten un marco adecuado para la enseñanza, y defienden la acción del docente y, más ampliamente, la investigación en didáctica como ámbito en el que avanzar.

Esperamos que el lector encuentre este contenido interesante.

\section{AgradeCimientos}

Las editoras encargadas de este número especial quieren dar las gracias a Xavier Fontich por la ayuda durante la preparación de este número temático. Igualmente, agradecen también a los 32 evaluadores y evaluadoras de 22 instituciones de 10 países su participación en la preparación de este monográfico:

Lourdes Aguilar (Universitat Autònoma de Barcelona), Elżbieta Awramiuk (Uniwersytet w Białymstoku), Marie-Claude Boivin (Université de Montréal), Víctor Corona Villavicencio (Universitat Autònoma de Barcelona), Ana Luísa Costa (Instituto Politécnico de Setúbal), Anna Czura (Uniwersytet Wrocławski), Kristin Denham (Western Washington University), Ilse Depraetere (Université de Lille), Carme Durán Rivas (Universitat Autònoma de Barcelona), Marie-Laure Elalouf (Université de Cergy-Pontoise), Santiago Fabregat Barrios (Universidad de Jaén), Xavier Fontich (Universitat Autònoma de Barcelona), María José García Folgado (Universitat de València), Maria Dolores Garcia-Pastor (Universitat de València), Mabel Giammatteo (Universidad de Buenos Aires), Patrice Gourdet (Université de Cergy-Pontoise), Edita Gutiérrez Rodríguez (Universidad Complutense de Madrid), Daniel Karczewski (Uniwersytet w Białymstoku), Wonki Lee (Yonsei 
University), Véronique Marmy Cusin (Haute École Pédagogique Fribourg), Sandra Martin-Chang (Concordia University), Beatriz Martín Marchante (Universitat Politècnica de València), Emilee Moore (Universitat Autònoma de Barcelona), Dave Pippin (Young Achievers Science and Math Pilot School), Abelard Saragossà (Universitat de València), Eli Sears (Portland State University), Liane She Honeycutt (Stanly Community College), Carmen Rodríguez Gonzalo (Universitat de València), Jean-Pierre Sautot (Université Claude Bernard), Carmen Sotomayor (Universidad de Chile), Marcial Terrádez Gurrea (Universitat de València) y François Vincent (Université du Québec en Outaouais).

\section{REFERENCIAS}

Bronckart, J.-P. y Besson, M.-J. (1988). Et si la grammaire n'était pas inutile? En D. Bain (Ed.), La recherche au service de l'enseignement? (pp. 73-93). Ginebra: CRPP. https://archive-ouverte.unige.ch/unige: 37612

Camps, A. y Milian, M. (2020). Metalinguistic activity in learning to write. En A. Camps y X. Fontich (Eds.), Research and teaching at the intersection: Navigating the territory of grammar and writing in the context of metalinguistic activity (pp. 123-144). Bruselas: Peter Lang.

Chartrand, S.-G. (2012). Quelles finalités pour l'enseignement grammatical à l'école? Une analyse des points de vue des didacticiens du français depuis 25 ans. Formation et profession, 20(3), 48-59. http://dx.doi.org/10.18162/fp.2012.222

Combettes, B. (1982). Grammaire et enseignement du français. Pratiques: linguistique, littérature, didactique, 33, 3-11. https://doi.org/10.3406/prati.1982.1227

Fontich, X. y García-Folgado, M. J. (2018). Grammar instruction in the Hispanic area: The case of Spain with attention to empirical studies on metalinguistic activity. Contribution to the special issue Working on grammar at school in L1 education: Empirical research across linguistic regions. L1-Educational Studies in Language and Literature, 18, 1-39. https://doi.org/10.17239/L1ESLL-2018.18.04.02

Hudson, R. (2016). Grammar instruction. En C. MacArthur, S. Graham y J. Fitzgerald (Eds.), Handbook of writing research (pp. 288-300). Nueva York, Londres: Guilford.

Lefrançois, P. y Brissaud, C. (2015). Les exigences linguistiques de quelques systèmes scolaires: une comparaison internationale. Mesure et évaluation en éducation, 38(3), 123-151. https://doi.org/10.7202/1036701ar

Milian, M. (2020). The instructional sequence model, twenty years on: A valid model for teaching how to learn and learning how to teach. En A. Camps y X. Fontich (Eds.), Research and teaching at the intersection: Navigating the territory of grammar and writing in the context of metalinguistic activity (pp. 341-350). Bruselas: Peter Lang.

Myhill, D., Watson, A. y Newman, R. (2020). Thinking differently about grammar and metalinguistic understanding in writing. Bellaterra Journal of Teaching \& Learning Language \& Literature, 13(2), e870. https://doi.org/10.5565/rev/jt13.870

Nadeau, M. y Fisher, C. (2006). La grammaire nouvelle. La comprendre et l'enseigner. Montreal: Gaëtan Morin.

Rodríguez-Gonzalo, C. y Zayas, F. (2017). La relación entre conocimientos gramaticales y el aprendizaje de prácticas discursivas: El adjetivo como ejemplo. Caplletra, 63, 245-277. https://doi.org/10.7203/caplletra.63.10401 
Schneuwly, B. (1998). Tout n'est pas métalangagier dans l'enseignement du français. La grammaire doit-elle être utile? En J. Dolz y J.-C. Meyer (Eds.), Activités métalangagières et enseignement du français. Actes des journées d'étude en didactique du français (Cartigny, 28 février-1 mars 1997) (pp. 267-272). Bruselas: Peter Lang. https://archive-ouverte.unige.ch/unige:31420

Vincent, F., Émery-Bruneau, J., Dezutter, O., Lefrançois, P. y Larose, F. (2016). L'enseignement de la grammaire au service du développement de compétences en lecture et en écriture : une synthèse des connaissances. Rapport de recherche. Québec: Fonds de recherche du Québec - Société et culture. http://www.frqsc.gouv.qc.ca/docuents/11326/2510085/PT_VincentF_rapport_synthese-connaissances.pdf/0701273172f1-48fa-92d2-571c5d09bd2b

Zayas, F. (2020). The composition of the news. En A. Camps y X. Fontich (ed.), Research and teaching at the intersection: Navigating the territory of grammar and writing in the context of metalinguistic activity (p. 101-106). Bruselas: Peter Lang.

\section{SYLVIE MARCOTTE}

Profesora en el Departamento de Ciencias de la Educación de la Université du Québec en Outaouais, enseña didáctica del francés como primera lengua. Su investigación aborda la enseñanza y el aprendizaje de la escritura desde la escuela primaria hasta la universidad, así como los vínculos entre las estrategias de enseñanza y el aprendizaje de los alumnos.

sylvie.marcotte@uqo.ca https://orcid.org/0000-0002-6623-6169

\section{MORGANE BEAUMANOIR-SECQ}

Doctora en Ciencias del Lenguaje (Université de Cergy Pontoise, 2016) y profesora de Ciencias de la Educación (Université de Paris), trabaja en el campo de la didáctica del francés, incidiendo en las representaciones de los estudiantes de primaria sobre el sistema sintáctico y en las cuestiones planteadas por la terminología gramatical.

morgane.beaumanoir-secq@u-paris.fr https://orcid.org/0000-0001-6528-0806

\section{AINA REIG}

Doctora en Didáctica de la Lengua por la Universitat de València (2020) y miembro del grupo de investigación GIEL de esta misma universidad. Ha sido docente en la Université de Picardie Jules Verne y en la Université Sorbonne Nouvelle de París. Su línea de investigación se centra en la gestión informativa en el proceso de composición escrita en colaboración.

ainareig@gmail.com https://orcid.org/0000-0001-5202-6876

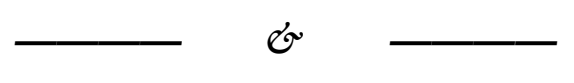




\title{
NOTE DES ÉDITRICES : RECHERCHE ACTUELLE SUR L'ENSEIGNEMENT DE LA GRAMMAIRE. POURQUOI ENSEIGNER LA GRAMMAIRE ?
}

\begin{abstract}
Résumé : Les recherches sur l'enseignement de la grammaire portent sur des thèmes variés et adoptent des perspectives plurielles. La III Conférence internationale sur l'enseignement de la grammaire (Congram19), tenue à l'Université autonome de Barcelone du 23 au 25 janvier 2019, en est une démonstration. Le côtoiement de communications portant sur des recherches en cours menées dans des contextes multiples a été l'occasion de consolider un champ commun dans lequel il est possible de débattre, tout comme de réfléchir aux particularités de la recherche menée dans chacun de ces contextes, liées notamment aux finalités attribuées à l'enseignement de la grammaire. Dans ce numéro spécial sont rassemblées les contributions de 16 chercheurs qui découlent de ce congrès. Le lecteur trouvera ces contributions en deux parties: la première dans le dernier numéro du Bellaterra Journal of Teaching \& Learning Language \& Literature (13.2) et la deuxième dans le présent numéro (13.3).
\end{abstract}

Mots-clés : Congram19 ; Enseignement de la grammaire ; Enseignement primaire ; Enseignement secondaire ; Enseignement supérieur

À la mémoire de Wayne O’Neil (1931-2020)

Participer à des évènements scientifiques internationaux comme la $I I I^{e}$ Conférence internationale sur l'enseignement de la grammaire (Congram19), tenue à l'Université autonome de Barcelone du 23 au 25 janvier 2019, représente, pour les jeunes chercheuses que nous sommes, une formidable occasion de prendre connaissance de recherches sur l'enseignement de la grammaire menées actuellement dans des contextes multiples et avec des perspectives plurielles. Nous retenons de cet évènement en particulier qu'il existe bel et bien, à l'international, un champ commun dans lequel il est possible de discuter de l'enseignement de la grammaire. Ce genre d'évènement permet aussi de réfléchir aux enjeux propres à chaque contexte de recherche et d'enseignement. Au fil des communications scientifiques du Congram19, tout comme des articles qui en découlent, il nous est apparu que les attentes vis-à-vis de l'étude de la langue ne sont pas les mêmes dans tous les contextes et que la question des finalités attribuées à l'enseignement de la grammaire a une influence déterminante sur cet enseignement : la nature du défi linguistique que constitue le lire-écrire, le rapport sociétal à la norme, la tradition scolaire sont autant de paramètres qui influencent la place dévolue à la grammaire dans l'enseignement ainsi que les objets et les stratégies d'enseignement de la grammaire qui sont privilégiés.

Si l'on se situe dans le contexte francophone, les didacticiens du français sont loin d'un consensus sur la question des finalités de l'enseignement de la grammaire. Dans les années 1980, Combettes (1982) pointe les tensions qui traversent 
la question des finalités de l'enseignement de la langue, et Bronckart et Besson (1988) se demandent «Et si la grammaire n'était pas inutile ? ». Chartrand (2012) fait ressortir trois points de vue défendus dans les écrits en didactique du français ces 25 dernières années : le point de vue utilitariste, selon lequel la grammaire sert le développement des compétences langagières des élèves, ce qui repose sur le postulat que la grammaire aide les élèves à comprendre et à produire des discours oraux et, surtout, écrits; le point de vue communicationnel, selon lequel la grammaire est au service des pratiques de communication; le point de vue disciplinaire et culturel, selon lequel la grammaire est une discipline qui peut être enseignée pour elle-même, pour connaitre et comprendre le système et le fonctionnement de la langue. Le point de vue utilitariste est bien présent dans le contexte francophone, notamment au Québec (p. ex. Nadeau et Fisher, 2006; Vincent, Émery-Bruneau, Dezutter, Lefrançois et Larose, 2016).

Cela n'est assurément pas étranger avec le fait qu'en contexte francophone, on accorde une grande importance au respect des normes et conventions de la langue écrite, en d'autres mots à l'absence d'erreurs, surtout d'orthographe; la grammaire est alors vue comme un moyen d'éviter ces erreurs. À titre d'exemple, Lefrançois et Brissaud (2015), qui comparent les exigences linguistiques de différents systèmes scolaires, montrent qu'au Canada, dans le système scolaire ontarien anglophone, un élève est réputé maitriser la langue écrite quand les erreurs qu'il commet dans son texte ne nuisent pas à la clarté du sens à communiquer, alors que dans le système scolaire québécois francophone, il est réputé la maitriser quand le nombre d'erreurs linguistiques qu'il commet se situe sous un seuil donné, ce seuil étant d'ailleurs plus strict au Québec que dans d'autres systèmes scolaires francophones (Nouveau-Brunswick, Belgique). Toujours dans le contexte francophone, le point de vue disciplinaire et culturel, selon lequel l'enseignement de la grammaire ne doit pas nécessairement être au service du développement des compétences langagières des élèves, est aussi présent. Parmi d'autres, Schneuwly (1998) considère que la grammaire peut être enseignée pour faire comprendre le fonctionnement de la langue, faire connaitre ses régularités et la faire percevoir comme un système; elle peut aussi l'être pour développer une attitude de découverte par rapport à celle-ci, voire développer des habiletés cognitives en général. Comme l'explique Chartrand (2012), selon ce point de vue, le travail grammatical permet aux élèves de transformer leur rapport à la langue, alors devenue un objet d'analyse, et donc de mettre celle-ci à distance, d'entrer dans un mode de pensée distinct et de se forger une culture grammaticale. Enseigner la grammaire aide ainsi le développement intellectuel et culturel des élèves. 
Si l'on se situe maintenant dans le contexte hispanique, ces 25 dernières années, un débat a aussi cours à propos des finalités de l'enseignement de la grammaire (voir Fontich et Garcia Folgado, 2018). En Espagne, dans les années 90, des approches communicatives sont mises de l'avant. La grammaire formelle cesse d'être la référence et l'enseignement de la grammaire est subordonné à l'enseignement de la langue d'usage. Dans la région de Valence, un programme innovant est développé sur la base des théories sociodiscursives et des genres discursifs; la grammaire est enseignée dans des tâches de communication orale, de lecture et d'écriture (voir p. ex. Zayas, 2020). La recherche montre ensuite que les approches communicatives doivent être combinées avec une approche plus explicite et systématique afin de doter les élèves de connaissances grammaticales organisées en un système cohérent, ce qui est impossible en enseignant la grammaire uniquement de manière accessoire en contexte de la communication. Les recherches basées sur l'interprétation de la réalité complexe de la classe et des processus d'enseignement et d'apprentissage qui s'y déroulent qui sont menées notamment en Catalogne et à Valence montrent le rôle central de l'activité métalinguistique dans l'enseignement des langues (Camps et Milian, 2020). Elles montrent également que cette activité métalinguistique, source intarissable d'apprentissage grammatical, peut être générée quand les élèves écrivent, collaborent ou font de la grammaire pour elle-même. Un modèle d'enseignement s'inscrivant dans cette perspective est celui des "séquences didactiques d'apprentissage de la grammaire »(Milian, 2020) ; il s'agit de projets qui comprennent des activités d'observation, de manipulation et d'analyse de formes linguistiques s'inscrivant à la fois à l'intérieur et à l'extérieur de situations de communication et qui mettent l'accent sur la relation entre les concepts grammaticaux (p. ex. Rodríguez-Gonzalo et Zayas, 2017). Ces séquences d'enseignement proposent trois approches différentes de l'apprentissage de la grammaire : l'étude de la langue dans les usages communicatifs, l'exploration des concepts du système grammatical et le contraste entre les langues, ou dialectes ou registres d'une même langue (Milian, 2020).

Pour des chercheuses évoluant dans le contexte francophone, dans lequel dominent les points de vue utilitariste et, dans une moindre mesure, disciplinaire et culturel, assister à un congrès comme le Congram19 est l'occasion de voir comment est actualisée et enseignée la grammaire dans des contextes où les finalités de cet enseignement sont autres, comme les contextes anglophone et hispanique. Pensons par exemple, dans le contexte anglophone, à la contribution de Myhill, Watson et Newman (2020), dans la première partie de ce numéro spécial (numéro 13.2) : les chercheuses défendent la pertinence de s'inscrire dans une perspective où la 
grammaire permet de faire des choix parmi un large éventail de ressources linguistiques et de prendre conscience des effets de ces choix chez le lecteur. En contexte anglophone, dans lequel un accent moins grand est mis sur le respect des normes linguistiques, une telle perspective de l'enseignement de la grammaire, communicationnelle, peut prendre plus de place qu'en contexte francophone. Cela étant dit, certains défendent tout de même, en contexte anglophone, que la grammaire doive être enseignée pour elle-même. Par exemple, Hudson (2016) considère que «Si la grammaire est seulement enseignée quand elle est directement liée à une tâche d'écriture, alors il n'y a essentiellement aucun enseignement systématique de la grammaire " (notre trad., p. 293) et émet une mise en garde à l'effet que cela peut avoir comme conséquence que les apprentissages grammaticaux prennent la forme d'« une liste non structurée d'éléments non liés » (notre trad., p. 293). Pour une chercheuse évoluant dans le contexte hispanique, dans lequel dominent les points de vue communicationnel et disciplinaire, le Congram19 est l'occasion de voir par exemple qu'en contexte francophone, la norme a encore une grande place dans l'enseignement grammatical et que l'écriture collaborative y est aussi vue comme une façon de générer une activité métalinguistique chez les élèves.

C'est pour continuer à consolider un champ commun dans lequel il est possible de débattre et à réfléchir aux particularités de la recherche menée dans plusieurs contextes que nous présentons ce numéro spécial du Bellaterra Journal of Teaching \& Learning Language \& Literature, avec 11 contributions (3 articles invités de chercheurs séniors, 6 articles de jeunes chercheurs, 2 entrevues et 3 comptes rendus) qui ont pour origine la $I I I^{e}$ Conférence internationale sur l'enseignement de la grammaire (Congram19). Les professeurs invités ont été conférenciers lors de trois des conférences plénières du Congram19 : Debra Myhill (Université d'Exeter), Ignacio Bosque (Université complutense de Madrid et Real Academia Española) et Enrique Bernárdez (Université complutense de Madrid). En ce qui concerne les entrevues, elles ont été recueillies auprès de quatre des chercheurs qui ont participé aux deux tables rondes organisées au cours de la conférence : Anna Camps (Université autonome de Barcelone), Maria Josep Cuenca (Université de Valence), Joaquim Dolz (Université de Genève) et Carmen Rodríguez-Gonzalo (Université de Valence). Ce sont, dans tous les cas, des chercheurs de renommée internationale qui, avec des perspectives diverses, ont apporté des contributions fondamentales au champ de recherche.

De plus, font partie de ce numéro spécial les articles de sept jeunes chercheurs, dont les recherches portent sur des contextes pluriels : l'enseignement primaire (Arseneau, Giguère), l'enseignement secondaire (Arseneau, Marcotte, Reig, 
Tormo) et l'enseignement supérieur (She Honeycutt et Sears), l'enseignement de la L1 (catalan, espagnol et français) et de la L2 (espagnol), les contextes monolingue (Arseneau, Giguère, Marcotte), bilingue (Reig, Tormo) et plurilingue (She Honeycutt et Sears), etc. Cette pluralité trouve aussi écho dans les différents rôles que peut jouer la grammaire, la place qui y est dévolue dans l'enseignement (grammaire liée à l'enseignement de la lecture et de l'écriture, grammaire liée au développement des compétences cognitives, grammaire enseignée pour elle-même) et par l'élément du triangle didactique qui est exploré : les apprenants et les processus d'apprentissage (Arseneau, Reig, Tormo), les enseignants et leurs connaissances et croyances, leurs pratiques, leurs méthodes et stratégies d'enseignement (Giguère, Marcotte, She Honeycutt et Sears) et, enfin, la grammaire comme objet d'enseignement (Arseneau, Marcotte, She Honeycutt et Sears).

Les contributions de ce numéro montrent que les recherches sur l'enseignement et l'apprentissage de la grammaire portent sur des thèmes variés et adoptent des perspectives plurielles. Le lecteur trouvera ces contributions en deux parties : la première partie dans le dernier numéro du Bellaterra Journal of Teaching \& Learning Language \& Literature (13.2) et la deuxième partie dans le présent numéro (13.3).

Nous présentons ci-dessous la deuxième partie, qui s'ouvre sur l'article d'un chercheur invité, celui du professeur Enrique Bernárdez (Université complutense de Madrid). Ce dernier s'interroge sur les objectifs de l'enseignement de la grammaire et sur les modèles qui prévalent à l'école et propose de concevoir l'enseignement de la grammaire comme une activité socioculturelle liée aux usages ( Enseñanza de la gramática como enseñanza de una actividad sociocultural »). Sa réflexion prend en compte les notions de langue d'usage et de langue standard, de pluricentrisme, ainsi que celles de variété et de registres linguistiques.

Le numéro se poursuit avec les contributions de trois jeunes chercheuses. Marie-Hélène Giguère ( «Le cercle pédagogique : pour développer les savoirs et les pratiques d'enseignement en grammaire actuelle») décrit les effets d'un dispositif de développement professionnel, le «cercle pédagogique ». Elle montre que les enseignants qui ont participé à des cercles pédagogiques pendant un an ont développé des pratiques pédagogiques et des savoirs liés à la grammaire actuelle et à son enseignement. Aina Reig ( Regulación metacognitiva y actividad metalingüística durante el proceso de composición escrita en colaboración a partir de la lectura de fuentes diversas ") analyse une intervention didactique dans laquelle des élèves du secondaire devaient rédiger en collaboration un texte en langue catalane. L'analyse 
des interactions et des brouillons des élèves montre les différences dans le raisonnement des élèves ainsi que l'appropriation de certaines des stratégies de régulation des contenus prévues dans l'intervention didactique. Enfin, Rosianne Arseneau ( «Comment la ponctuation se développe-t-elle chez les élèves ? Une revue systématique des recherches empiriques en classe de français langue d'enseignement») a analysé 15 études empiriques du contexte francophone portant sur le développement de la ponctuation à l'école primaire et secondaire. Elle propose trois grands stades d'acquisition de la ponctuation et souligne la nécessité de mener des recherches qui mettent en relation des interventions didactiques menées en classe et les apprentissages des élèves.

Finalement, ce numéro se clôt par un compte rendu d'ouvrage et une entrevue. Anabella L. Poggio (Université de Buenos Aires) fait le compte rendu d'un ouvrage portant sur les recherches menées au cours des 25 dernières années par des chercheurs de l'Université autonome de Barcelone, hôte du Congram19. Cet ouvrage, dirigé par Anna Camps et Xavier Fontich, a été publié simultanément en anglais (Research and teaching at the intersection: Navigating the territory of grammar and writing in the context of metalinguistic activity, Peter Lang, 2020) et en espagnol (La actividad metalingüística como espacio de encuentro de la escritura y la gramática: Un itinerario de enseñanza e investigación en educación lingüística, Universidad Nacional de San Juan, 2020) et la version en espagnol est disponible en ligne en libre accès. Dans "The role of grammar teaching in learning to write ", Joaquim Dolz (Université de Genève) et Carmen Rodríguez-Gonzalo (Université de Valence) accordent un entretien à Alba Compte. Ils parlent des ponts entre la grammaire et l'écriture et de la révision comme déclencheur de la réflexion sur la langue. Dolz et Rodríguez-Gonzalo considèrent que la relation entre grammaire et écriture ne peut être construite sur la base d'un travail accessoire sur la grammaire mené en contexte d'écriture. Ils remettent aussi en question les recherches qui reposent sur un cadre strictement linguistique pour penser l'enseignement et défendent la pertinence des recherches centrées sur l'action de l'enseignant et, plus largement, la recherche en didactique.

Nous espérons que vous trouverez la deuxième partie de ce numéro spécial intéressante. 


\section{REMERCIEMENTS}

Les éditrices invitées tiennent à remercier Xavier Fontich pour son aide dans la préparation de ce numéro. Elles remercient également les 32 évaluateurs de 22 institutions et de 10 pays qui ont participé à la préparation de ce numéro :

Lourdes Aguilar (Universitat Autònoma de Barcelona), Elżbieta Awramiuk (Uniwersytet w Białymstoku), Marie-Claude Boivin (Université de Montréal), Víctor Corona Villavicencio (Universitat Autònoma de Barcelona), Ana Luísa Costa (Instituto Politécnico de Setúbal), Anna Czura (Uniwersytet Wrocławski), Kristin Denham (Western Washington University), Ilse Depraetere (Université de Lille), Carme Durán Rivas (Universitat Autònoma de Barcelona), Marie-Laure Elalouf (Université de Cergy-Pontoise), Santiago Fabregat Barrios (Universidad de Jaén), Xavier Fontich (Universitat Autònoma de Barcelona), María José García Folgado (Universitat de València), Maria Dolores Garcia-Pastor (Universitat de València), Mabel Giammatteo (Universidad de Buenos Aires), Patrice Gourdet (Université de Cergy-Pontoise), Edita Gutiérrez Rodríguez (Universidad Complutense de Madrid), Daniel Karczewski (Uniwersytet w Białymstoku), Wonki Lee (Yonsei University), Véronique Marmy Cusin (Haute École Pédagogique Fribourg), Sandra Martin-Chang (Concordia University), Beatriz Martín Marchante (Universitat Politècnica de València), Emilee Moore (Universitat Autònoma de Barcelona), Dave Pippin (Young Achievers Science and Math Pilot School), Abelard Saragossà (Universitat de València), Eli Sears (Portland State University), Liane She Honeycutt (Stanly Community College), Carmen Rodríguez Gonzalo (Universitat de València), Jean-Pierre Sautot (Université Claude Bernard), Carmen Sotomayor (Universidad de Chile), Marcial Terrádez Gurrea (Universitat de València) et François Vincent (Université du Québec en Outaouais).

\section{RÉFÉRENCES}

Bronckart, J.-P. et Besson, M.-J. (1988). Et si la grammaire n'était pas inutile ? Dans D. Bain (dir.), La recherche au service de l'enseignement? (p. 73-93). CRPP. https://archive-ouverte.unige.ch/unige:37612

Camps, A. et Milian, M. (2020). Metalinguistic activity in learning to write. Dans A. Camps et X. Fontich (dir.), Research and teaching at the intersection: Navigating the territory of grammar and writing in the context of metalinguistic activity (p. 123-144). Peter Lang.

Chartrand, S.-G. (2012). Quelles finalités pour l'enseignement grammatical à l'école ? Une analyse des points de vue des didacticiens du français depuis 25 ans. Formation et profession, 20(3), 48-59. http://dx.doi.org/10.18162/fp.2012.222

Combettes, B. (1982). Grammaire et enseignement du français. Pratiques : linguistique, littérature, didactique, 33, 3-11. https://doi.org/10.3406/prati.1982.1227 
Fontich, X. et García-Folgado, M. J. (2018). Grammar instruction in the Hispanic area: The case of Spain with attention to empirical studies on metalinguistic activity. Contribution to the special issue Working on grammar at school in L1 education: Empirical research across linguistic regions. L1-Educational Studies in Language and Literature, 18, 1-39. https://doi.org/10.17239/L1ESLL-2018.18.04.02

Hudson, R. (2016). Grammar instruction. Dans C. MacArthur, S. Graham et J. Fitzgerald (dir.), Handbook of writing research ( $2^{\mathrm{e}}$ éd.) (p. 288-300). Guilford.

Lefrançois, P. et Brissaud, C. (2015). Les exigences linguistiques de quelques systèmes scolaires : une comparaison internationale. Mesure et évaluation en éducation, 38(3), 123-151. https://doi.org/10.7202/1036701ar

Milian, M. (2020). The instructional sequence model, twenty years on: A valid model for teaching how to learn and learning how to teach. Dans A. Camps et X. Fontich (dir.), Research and teaching at the intersection: Navigating the territory of grammar and writing in the context of metalinguistic activity (p. 341-350). Peter Lang.

Myhill, D., Watson, A. et Newman, R. (2020). Thinking differently about grammar and metalinguistic understanding in writing. Bellaterra Journal of Teaching \& Learning Language \& Literature, 13(2), e870. https://doi.org/10.5565/rev/jt13.870

Nadeau, M. et Fisher, C. (2006). La grammaire nouvelle. La comprendre et l'enseigner. Gaëtan Morin.

Rodríguez-Gonzalo, C. et Zayas, F. (2017). La relación entre conocimientos gramaticales y el aprendizaje de prácticas discursivas: El adjetivo como ejemplo. Caplletra, 63, 245-277. https://doi.org/10.7203/caplletra.63.10401

Schneuwly, B. (1998). Tout n'est pas métalangagier dans l'enseignement du français. La grammaire doit-elle être utile ? Dans J. Dolz et J.-C. Meyer (dir.), Activités métalangagières et enseignement du français. Actes des journées d'étude en didactique du français (Cartigny, 28 février-1 mars 1997) (p. 267-272). Peter Lang.

https://archive-ouverte.unige.ch/unige: 31420

Vincent, F., Émery-Bruneau, J., Dezutter, O., Lefrançois, P. et Larose, F. (2016). L'enseignement de la grammaire au service du développement de compétences en lecture et en écriture : une synthèse des connaissances. Rapport de recherche. Fonds de recherche du Québec Société et culture.

http://www.frqsc.gouv.qc.ca/documents/11326/2510085/PT_VincentF_rapport_syntheseconnaissances.pdf/07012731-72f1-48fa-92d2-571c5d09bd2b

Zayas, F. (2020). The composition of the news. Dans A. Camps et X. Fontich (dir.), Research and teaching at the intersection: Navigating the territory of grammar and writing in the context of metalinguistic activity (p. 101-106). Peter Lang.

\section{SYLVIE MARCOTTE}

Professeure au Département des sciences de l'éducation de l'Université du Québec en Outaouais, elle enseigne la didactique du français langue première. Ses recherches touchent l'enseignement et l'apprentissage de l'écrit du primaire à l'université ainsi que les liens entre les stratégies pédagogiques et les apprentissages des élèves. 


\section{MORGane BEAUMANOIR-SECQ}

Docteure en sciences du langage (Université de Cergy-Pontoise, 2016) et maitre de conférences en Sciences de l'Éducation (Université de Paris), elle travaille dans le champ de la didactique de l'étude de la langue en français, s'intéressant notamment aux représentations des élèves du primaire sur le système syntaxique, mais aussi aux enjeux portés par la terminologie grammaticale.

morgane.beaumanoir-secq@u-paris.fr https://orcid.org/0000-0001-6528-0806

\section{AINA REIG}

Docteure en didactique des langues (Universitat de València, 2020) et membre du groupe de recherche GIEL de cette université, elle a été enseignante à l'Université de Picardie Jules Verne et à l'Université Sorbonne Nouvelle à Paris. Ses recherches portent notamment sur la gestion de l'information dans le processus de production écrite en collaboration.

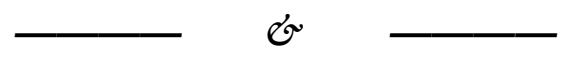

Marcotte, S., Beaumanoir-Secq, M., \& Reig, A. (2020). Editors' Note: Current Research on Grammar Teaching. What is the Use of Teaching Grammar? Bellaterra Journal of Teaching \& Learning Language \& Literature, 13(3), e966. https://doi.org/10.5565/rev/jtl3.966 